\title{
MAJOR OBSTETRICAL COMPLICATIONS IN AN ELDERLY PRIMIPAROUS WITH A MULTITUDE OF PRE-EXISTING PATHOLOGIES
}

\author{
Vasiliu Cristina ${ }^{1,2}$, Cojocaru Lorena ${ }^{1}$, Malusanu Patricia ${ }^{1}$, Albu Elena Simona ${ }^{2}$, Cîrstoiu Monica ${ }^{1,2}$ \\ ",Carol Davila” University of Medicine and Pharmacy, Bucharest, Romania \\ ${ }^{2}$ Emergency University Hospital of Bucharest, Romania
}

\section{CASE Abstract REPORT}

DOI: $10.33695 /$ rojes.v2i1.24

Accepted: 10.04 .2020

The number of primiparous pregnant women, over the age of 35 , has increased considerably in the past few years, because of the elevated level of education and also because the multitude of contraceptive methods. Such a patient suits the category of elderly primiparous and it represents a real obstetrical challenge, due to the numerous complications related to pregnancy and the immediate postpartum, as well as those related to the neonatal prognosis. We present a 35 old years patient, second gestation, primiparous, a partially monitored pregnancy, known with essential hypertension grade III ESC / ESA, insufficiently controlled therapeutically, with target organ damage (exudative retinal detachment, hypertensive choroidopathy), morbid obesity and also with the suspicion of a renal tumor. The rate of complications occurring in the case of an elderly primiparous was increased in the present case. Obstetrical complications occurred: preeclampsia, HELLP (hemolysis, elevated liver enzymes, low platelet count) syndrome, placental abnormalities (placenta accreta), intrauterine growth restriction, chronic fetal distress and finally fetal death in utero. The evolution of the patient in postpartum was burdened by the decompensation of the pre-existing pathology. In conclusion, elderly primiparous is susceptible to obstetrical complications during pregnancy and childbirth, such as premature birth, fetal acute distress, which may result in fetal death, placental abruption, placental abnormalities, preeclampsia, eclampsia, therefore following up the pregnancy requires increased attention and is also a real challenge for the obstetrician.

Corresponding author:

Albu Elena Simona

albuelenasimona@ gmail.com

Keywords: elderly primiparous, preeclampsia, fetal distress, increased morbidity and mortality

\section{Introduction}

The number of primiparous pregnant women, over the age of 35 , has increased considerably in the past few years, because of the elevated level of education and also by the multitude of contraceptive methods. Such a patient suits the category of elderly 
primiparous and is a real obstetrical challenge, because of the numerous complications related to pregnancy and the immediate postpartum, as well as those related to the neonatal prognosis [1].

The purpose of our paper is to draw attention to the serious evolution with an ominous prognosis that a pregnancy and childbirth can have in an elderly primiparous and obese woman with various pre-existing pathologies.

Among the most common maternal complications, we can mention, in order of frequency, anemia, preeclampsia, eclampsia, placental adhesion abnormalities, placental abruption, gestational diabetes, and an increased rate of births by cesarean section. Regarding fetal risks, cases of acute fetal distress, congenital anomalies, intrauterine growth restriction, increased risk of neonatal morbidity and mortality may occur [2], [3].

Thus, an elderly primiparous requires close monitoring, considering that it is a pregnancy with a major obstetrical risk. However, following-up such a pregnancy becomes even more difficult for the obstetrician, when other pathologies are associated that can influence the maternalfetal prognosis.

\section{Case presentation}

We will present case of a surgical and obstetrical emergency, a pregnant woman, 35 years old, secundigravida, primiparous, pregnant 30 weeks, with miscellaneous preexisting pathologies, who presented to our emergency department for absent active fetal movements, with ultrasound confirmation of fetal death in utero, on the same day, hypertensive burst (Blood Pressure $=180 / 100$ $\mathrm{mmHg}$ ), painful uterine contractions, black blood metrorrhagia, visual disorders which started two weeks before.

From the patient's history we noted LMP (last menstrual period)-13.09.2019, menarche 13 years, regular menstrual cycle, one miscarriage, pre-existing hypertension in treatment for 5 years, but insufficiently controlled therapeutically, with target organ damage, exudative retinal detachment and morbid obesity, affirmatively renal lithiasis, suspicion of renal tumor, of unspecified etiology, being insufficiently investigated.

The patient subjective accusations are: visual disorders and headache. At admission she is conscious, cooperative, with altered general condition and high blood pressure values $(180 / 100 \mathrm{mmHg})$. The abdomen is distended by the pregnant uterus. Gynecological examination reveals a minimal metrorrhagia and intact amniotic membranes. The cervix is slightly shortened, oriented posteriorly, the presentation is cranial, mobile, and uterine tonus is increased.

Paraclinical investigations performed at hospitalization indicate leukocytosis with neutrophilia $(11,000$ / $\mathrm{mm} 3)$, thrombocytopenia $(83,000$ / $\mathrm{mm} 3)$, hepatocytolysis with an increase in transaminases 4 times above normal, hyperuricemia, hemoglobin $11.3 \mathrm{~g} / \mathrm{dL}$.

Following the multidisciplinary evaluation in ER, the next stage diagnoses are established: IIGIP (secundigesta, primiparous) pregnancy 30 weeks, antepartum fetal death, severe preeclampsia, HELLP (hemolysis, elevated liver enzymes, low platelet count) syndrome, placental abruption, pre-existing hypertension grade III ESC / ESA, fluid pericarditis in minimal amount, detachment retinal exudation, hypertensive choroidopathy.

The patient is admitted in the Ob-Gyn department and an emergency cesareansection is performed for maternal purposes:

- Severe preeclampsia due to pre-existing hypertension in pregnancy

- HELLP syndrome

- Placental abruption

When opening the abdomen, free fluid in the peritoneal cavity was observed (approx. $300 \mathrm{ml})$. 
A segmental-transverse hysterotomy was performed, followed by the extraction of a dead, female fetus, weighing about $970 \mathrm{~g}$ and of a placental mass, weighing about $300 \mathrm{~g}$. An anterior hematoma of about $2 / 3 \mathrm{~cm}$ and an increased degree of placental adherence in the posterior region where observed. The cultures prelevated intraoperatory from the peritoneal fluid and from the uterine cavity were negative.

Postoperatively the patient is conscious, cooperative, afebrile, hemodynamically stable, but with a tendency to hypertension under antihypertensive treatment (alphametyldopa, amlodipine). Antibiotic, analgesic and anticoagulant treatments are continued.

The postoperative evolution is favorable, the uterus is physiologically retracted, the lochia appears normal, odorless, the postoperative wound is apparently supple. Subsequently, the evolution is complicated by high blood pressure values, difficult to control therapeutically and marked proteinuria, over $3 \mathrm{~g} / 1000 \mathrm{ml}$.

Therefore, the suspicion of secondary hypertension is raised and interdisciplinary consultations are requested: cardiology, nephrology, urology. In addition, abdominal ultrasound, abdomino-pelvic scans, CT and MRI are performed.

The scans indicate the presence of a space-replacing structure, located left perirenal, of approximately 73/53/100 mm, relatively plainly delimited by a pseudo capsule, without signs of active bleeding at its level - old hematoma, which refutes the differential diagnosis of Gravitz tumor, suspected in the beginning.

Maximal antihypertensive treatment was initiated with:

- Bisoprolol 5 mg 1-0-0

- Candesartan 16 mg 1-0-0

- Amlodipine 10 mg 1-0-0

- Alpha-metyldopa 250 mg 1-1-1 or 1-2-1 adjusted according to blood pressure values

The nephrologist recommends monitoring the diuresis / $24 \mathrm{~h}$, dosage of proteinuria and albuminemia / 24h, Addis test, sideremia, ferritin, alkaline phosphatase, total proteins, lipid profile. Considering the high blood pressure values, uncontrolled under maximum treatment and also dyslipidemia, hypoalbuminemia and marked proteinuria, a nephrotic syndrome is suspected. In this context, a possible secondary reno-parenchymal hypertension, consequence of a glomerulopathy is questioned. For this reason, it is decided to transfer the patient in the nephrology department.

The patient's evolution is complicated by a febrile access with respiratory distress syndrome, raising the suspicion of infection with SARS-Cov2 (negative Rt-PCR at admission). Due to the high blood pressure values (Systolic arterial pressure $=240$ $\mathrm{mmHg}$ ) and putting in balance the thrombotic risk and that of a potential hemorrhagic stroke, the anticoagulant treatment was interrupted.

At the same time, the postoperative wound is infected with methicillin-resistant Staphylococcus aureus and with inducible resistance to Clindamycin.

Hence, it is decided to transfer the patient in the ICU (Intensive Care Unit) department. The respiratory distress syndrome is accentuated. Thoracic CT scan confirms the clinical suspicion and reveals massive pulmonary thromboembolism involving the right pulmonary artery with extension to all right lobe arteries, with quasicomplete occlusive character. The condensation aspect of the lung parenchyma through densification areas in matte glass maintains the suspicion of SARS infection. Cov 2 (negative Rt-PCR in dynamics). Abdominal scan shows decreased dimension of the retroperitoneal hematoma. Broadspectrum antibiotic therapy, anticoagulant therapy, renal dialysis and mechanical ventilation by oro-tracheal intubation are initiated. 
The evolution is complicated by a pulmonary infection, with positive bacterial identification for Klebsiella spp, which requires the adjustment of antibiotic therapy according to the antibiogram.

Lochioculture becomes positive, with bacterial identification for Pseudomonas spp, Klebsiella pneumoniae ESBL. Given the unfavorable evolution, with biological changes that plead for sepsis, we decided to perform total hysterectomy.

Intraoperatively, free serosanguineous fluid (approx.200 ml) is found, with negative results for the bacterial examination. The uterus is involuted $10 / 6 / 4 \mathrm{~cm}$, the surgical incision with no sign of dehiscence.

The histopathological examination of the uterus describes limited areas of accrete placenta, multiple intravascular thrombosis and fibrin deposits on the inner surface of the uterine body.

Postoperatively important leukocytosis, anemia and thrombocytopenia are present.

Clinically the patient's condition is maintained critical because of ventilation disorders secondary to pulmonary thromboembolism.

Imagistic, it appears a normal posthysterectomy status with a significant reduction of the perirenal hematoma. Thoracic CT shows accentuation of the preexisting condensation with appearance of new areas of pulmonary condensation. It remains a high suspicion of SARS-CoV-2 infection which cannot be demonstrated by Rt-PCR.

The evolution is unfavorable, with aggravation. The hematology consultation establishes the diagnosis of heparin- induced thrombocytopenia. Multisystem organ failure appears, occurring exitus by irreversible cardio-respiratory arrest.

\section{Discussions}

The medical history of the pre-existing hypertension with the registration of high blood pressure values under treatment, both during and before pregnancy, predicts the installation of a severe preeclampsia [4],[5],[6].

In preeclampsia, the central element is represented by a defect in the placental process. The defect is based on a deficient trophoblastic invasion of the intima of the spiral arteries. Consequently, a diminished uterine vascular bed is formed, spiral arteries are of small caliber, reactive to various pressure stimuli in the maternal body which cannot ensure optimal utero-placental flow [7],[8].

Insufficient utero-placental flow leads in time to intrauterine growth restriction, with deviation of fetal metabolism to conditions of hypoxia. Oxygen is directed to the strictly oxygen-dependent organs (central nervous system, myocardium, adrenal glands), with peripheral vasoconstriction [9]. Complications are shown in Table 1 [7],[10].

\begin{tabular}{cc}
\hline $\begin{array}{c}\text { Maternal } \\
\text { complications } \\
\text { secondary to } \\
\text { preeclampsia }\end{array}$ & $\begin{array}{c}\text { Fetal } \\
\text { complications } \\
\text { secondary to } \\
\text { preeclampsia }\end{array}$ \\
\hline Eclampsia & $\begin{array}{c}\text { Intrauterine growth } \\
\text { restriction/chronic } \\
\text { fetal distress }\end{array}$ \\
\hline Placental abruptio & Fetal death in utero \\
\hline HELLP Sindrome & Early neonatal death \\
\hline Acute Kidney Failure & Prematurity \\
\hline Pulmonary acute edema & Acute fetal distress \\
\hline Cerebral & $\begin{array}{c}\text { Neurological } \\
\text { sequelae due to } \\
\text { cerebral hypoxia }\end{array}$ \\
\hline ischemia/hemorrhage & \\
\hline Liver subcapsular \\
hematoma
\end{tabular}

Table 1- Maternal and fetal complications secondary to preeclampsia

*The marked rows present signs and symptoms that are found in the case of our patient

The mechanisms of fetal compensation are overcome, ischemia becomes present in different territories and the final consequence is fetal death in utero [9][10]. Hypoxemia, 
respectively acidosis at the level of the uteroplacental interface, determines the activation of maternal mechanisms that aim to increase blood supply to the intervillary space, the spiral arteries respond by vasoconstriction, which leads to local ischemia.

Prolonged ischemia and stasis cause damage to the endovascular endothelium, with the appearance of microthrombosis, therefore areas of necrosis, hemorrhagicischemic areas and areas of placental abruption can occur [11].

In this case, the favoring factors of a severe form of preeclampsia are the preexisting hypertension refractory to treatment and the morbid obesity. Morbid obesity is also a predisposing factor for wound infections postoperatively and an additional risk factor for thrombotic events during pregnancy. It is known that the risk of thrombotic events is considered maximum during pregnancy and postpartum even in a pregnant woman without other associated pathologies [12],[13].

Referring to the present case, in addition to the general thrombotic risk associated with immediate postpartum, the following predisposing factors are added: chronic hypertension, morbid obesity, preeclampsia, nephrotic syndrome, cesarean delivery, age over 35 years, immobilization by prolonged bed rest, discontinuation of anticoagulant treatment (considering the riskbenefit ratio between the hemorrhagic risk under anticoagulant treatment vs. thrombotic risk) [14], [15].

The potentially aggravating element of the patient's evolution was represented by massive pulmonary thromboembolism complicated by bronchopneumonia with Klebsiella pneumoniae. Bronchopneumonia was favored by PET, pulmonary stasis, mechanical ventilation with IOT probe and thus was maintained a vicious circle [16].

Analyzing the case, we can establish as particularities the following aspects: severe preeclampsia added to pre-existing hypertension, advanced age at which the first pregnancy was obtained, morbid obesity, secondary hypertension, most likely of renoparenchymal cause (with suspected glomerulopathy) refractory to treatment, as well as the pulmonary imaging aspect that pleads for a SARS-CoV-2 infection, which cannot be demonstrated by Rt-PCR tests [17],[18].

\section{Conclusions}

Generally, following-up a pregnancy requires increased attention from both the obstetrician and the pregnant woman, especially in the case of a pregnancy obtained at an age of more than 35 years, when the risk of obstetrical complications is increased.

The real challenge arises, however, when in addition to the multitude of pathologies that can occur in an elderly primiparous, pre-existing maternal pathologies are added to the pregnancy.

In such situations, a good collaboration between the obstetrician, a multidisciplinary team and the pregnant woman must be developed. The patient must be advised to monitor the blood pressure at home, control the weight, follow the imposed treatment schemes and, of course, to immediately address to the medical services in case of alarm signals, such as: diminished active fetal movements, painful uterine contractions not responsive to lithic medication, increased uterine tonus, metrorrhagia, altered general condition.

\section{References}

[1] Morrison I. The elderly primigravida. Am J Obstet Gynecol. 1975;121(4):465-470. doi:10.1016/0002-9378(75)90076-9

[2] Sutton ALM, Harper LM, Tita ATN. Hypertensive Disorders in Pregnancy. Obstet Gynecol Clin North Am. 2018;45(2):333-347. doi:10.1016/j.ogc.2018.01.012 
Major obstetrical complications in an elderly primiparous with a multitude of pre-existing pathologies

[3] Jauniaux E, Dimitrova I, Kenyon N, et al. Impact of placenta previa with placenta accreta spectrum disorder on fetal growth. Ultrasound Obstet Gynecol. 2019;54(5):643649. doi:10.1002/uog.20244

[4] López-Carbajal MJ, Manríquez-Moreno ME, Gálvez-Camargo D, Ramírez-Jiménez E. Factores de riesgo asociados con preeclampsia [Risk factors associated to preclampsia]. Rev Med Inst Mex Seguro Soc. 2012;50(5):471-476.

[5] Brown MA, Magee LA, Kenny LC, et al. The hypertensive disorders of pregnancy: ISSHP classification, diagnosis \& management recommendations for international practice. Pregnancy Hypertens. 2018;13:291-310.

doi:10.1016/j.preghy.2018.05.004

[6] Olson-Chen C, Seligman NS. Hypertensive Emergencies in Pregnancy. Crit Care Clin. 2016;32(1):29-41. doi:10.1016/j.ccc.2015.08.006

[7] Williams Obstetrics, 24e. F. Gary Cunningham, Kenneth J. Leveno, Steven L. Bloom, Jodi S. Dashe, Barbara L. Hoffman, Brian M. Casey, Catherine Y. Spong

[8] Tratat de chirurgie editia a II-a, Obstetrica si Ginecologie, coordonator Prof. Dr. Gheorghe Peltecu

[9] Quibel T, Bultez T, Nizard J, Subtil D, Huchon C, Rozenberg P. Morts fœtales in utero [In utero fetal death]. J Gynecol Obstet Biol Reprod (Paris). 2014;43(10):883-907. doi:10.1016/j.jgyn.2014.09.018

[10] Bokslag A, van Weissenbruch M, Mol BW, de Groot CJ. Preeclampsia; short and long-term consequences for mother and neonate. Early Hum Dev. 2016;102:47-50. doi:10.1016/j.earlhumdev.2016.09.007

[11]Downes KL, Grantz KL, Shenassa ED. Maternal, Labor, Delivery, and Perinatal
Outcomes Associated with Placental Abruption: A Systematic Review. Am J Perinatol. 2017;34(10):935-957. doi:10.1055/s-0037-1599149

[12] Rana S, Lemoine E, Granger JP, Karumanchi SA. Preeclampsia: Pathophysiology, Challenges, and Perspectives [published correction appears in Circ Res. 2020 Jan 3;126(1):e8]. Circ Res. 2019;124(7):1094-1112. doi:10.1161/CIRCRESAHA.118.313276

[13] Kattah AG, Garovic VD. The management of hypertension in pregnancy. Adv Chronic Kidney Dis. 2013;20(3):229239. doi:10.1053/j.ackd.2013.01.014

[14] Dado CD, Levinson AT, Bourjeily G. Pregnancy and Pulmonary Embolism. Clin Chest Med. 2018;39(3):525-537. doi:10.1016/j.ccm.2018.04.007

[15] Tan JY. Thrombophilia in pregnancy. Ann Acad Med Singapore. 2002;31(3):328334.

[16] Robertson, Lindsay; Greer, Ian Thromboembolism in pregnancy, Current Opinion in Obstetrics and Gynecology: April 2005 - Volume 17 - Issue 2 - p 113-116 doi: 10.1097/01.gco.0000162177.30607.59

[17] Marchi J, Berg M, Dencker A, Olander EK, Begley C. Risks associated with obesity in pregnancy, for the mother and baby: a systematic review of reviews. Obes Rev. 2015;16(8):621-638. doi:10.1111/obr.12288 [18] Lai CC, Shih TP, Ko WC, Tang HJ, Hsueh PR. Severe acute respiratory syndrome coronavirus 2 (SARS-CoV-2) and coronavirus disease-2019 (COVID-19): The epidemic and the challenges. Int $\mathrm{J}$ Antimicrob Agents. 2020;55(3):105924. doi:10.1016/j.ijantimicag.2020.105924. 